\title{
Potentials of mean force for the exo and endo solvolysis of 2-norbornyl chloride in water and DMSO: A constrained molecular dynamics study ${ }^{\#}$
}

\author{
SUBODH C TIWARI, TIMIR HAJARI, ASHISH SHARMA and B L TEMBE* \\ Department of Chemistry, Indian Institute of Technology Bombay, Mumbai 400 076, India \\ e-mail: bltembe@chem.iitb.ac.in
}

\begin{abstract}
Constrained molecular dynamics is used to calculate the potentials of mean force for the exo and endo dissociation of 2-norbornyl chlorides in two solvents, namely water and dimethyl sulphoxide (DMSO). The gas phase potential energy curves for the 2-norbornyl chlorides are computed by ab initio methods and these curves are fitted into a 12-6-1 potential for use in classical molecular dynamics simulations. The gas phase potential energy curves indicate a greater barrier for the dissociation of the endo 2-norbornyl chloride (than the exo). The potentials of mean force (PMF) in DMSO and water indicate the locations and the magnitudes of the activation barriers in these solutions and are sensitive functions of the fitted gas phase potentials.
\end{abstract}

Keywords. Potentials of mean force (PMF); constrained molecular dynamics; endo and exo 2-norbornyl chloride dissociation.

\section{Introduction}

Kinetics for the solvolysis of endo and exo norbornyl derivatives has remained an active area for both experimental and theoretical investigation for more than half a century. ${ }^{1-12}$ Weinstein and Trifan studied the acetolysis of 2-norbornyl arenesulphonates ${ }^{2}$ and observed that the exo-isomers react much faster than the endo isomers. Both the isomers solely give the exo products. The exo solvolytic rates are larger than the endo rates by nearly a factor of 1000 which leads to a difference of $\sim 6 \mathrm{kcal} / \mathrm{mol}$ in the activation energies for the two transition states. ${ }^{3}$ Initially, the idea of nonclassical stabilization was introduced to interpret this observation. The existence of nonclassical structure for 2-norbornyl cation was confirmed by Olah and other groups by spectroscopic measurements. ${ }^{4}$ However, Brown questioned the arguments supporting non classical structures using various experimental factors ${ }^{5}$ and Schleyer has critically examined Brown's argument. ${ }^{6}$ Debate over the participation of non-classical ${ }^{7}$ or classical cation ${ }^{8}$ has continued for a long time. Several computational studies show that the classical cation is more stable than non-classical one in water while in gas phase or in less polar solvent, non-classical form is expected to be more stable. ${ }^{9}$ Recently, an ion-pair leakage ('inter-conversion') from classical 2-norbornyl ion pair species to a non-classical one was introduced to rationalize the D-label scrambling observed

\footnotetext{
\#Dedicated to Prof. N Sathyamurthy on his 60th birthday

*For correspondence
}

experimentally in $\mathrm{DCl}$ addition products of nortricyclene and norbornene. ${ }^{10}$ In a related QTAIM-DI-VISAB study of 7-norbornyl cation, Werstiuk showed that this cation exhibits the molecular graph of the bicyclo[3.2.0]heptyl cation at its equilibrium geometry. ${ }^{11}$ In fragmentation patterns of related compounds such as exo and endo 2-norbornyloxy-chlorocarbenes, products included exo-norbornyl chloride, endo-norbornyl chloride, norbornene and nortricyclene. Fragmentation activation energies were very low $(\sim 4 \mathrm{kcal} / \mathrm{mol})$ and, as a result, rate constants for fragmentation were nearly identical for exo and endo. Due to the return of chloride within the ion pairs, more endo-chloride was formed from the endo-carbene. ${ }^{12}$

The above studies indicate that the detailed analysis of the solvent contribution to the dissociation process of the endo and the exo 2-norbornyl chlorides will help considerably in understanding the solvolytic processes in solution. For this purpose, we have considered the dissociation process of exo and endo 2-norbornyl chlorides in the gas phase [structures I and II shown in equation (1)] and in solution.

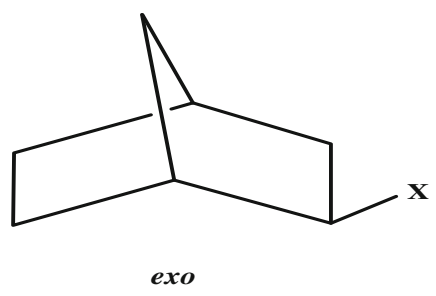

I

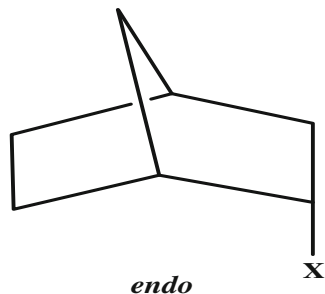

II 
In section 2, we outline the methodology used in our work. The results and discussions are given in section 3 and conclusions in section 4 .

\section{Methodology}

Our methodology has three main sections, (i) study of the gas phase dissociation of exo and endo 2-norbornyl chloride using $a b$ initio calculations. (ii) Parameterization of the potential energy functions for molecular dynamics simulations and (iii) molecular dynamics simulations for both endo and exo norbornyl chlorides.

$A b$ initio calculations were performed using quantum chemical program Gaussian 09. ${ }^{13}$ Norbornyl chloride is initially optimized in the gas phase at Hartree Fock level (HF), density functional (B3LYP), ${ }^{14}$ and the MP2 method. ${ }^{15} 6-311++\mathrm{G}^{* *}$ basis set was used for the calculations. The optimized geometries were further characterized by evaluating the vibrational frequencies at respective levels of theory. Rigid scan was performed using optimized geometries in the $\mathrm{C}-\mathrm{Cl}$ direction to obtain potential energy curves for dissociation of endo and exo 2-norbornyl chlorides. Potential energy curves obtained from the $a b$ initio calculation at B3LYP/6$311++\mathrm{G}^{* *}$ is used for the parameterization of the potential energy. The $\mathrm{C}-\mathrm{Cl}$ distance is effectively the reaction coordinate for the dissociation for both the exo and endo norbornyl chlorides.

The molecules under investigation are confined in a cubical box in the MD simulations. The initial configurations and velocities of the molecules are specified. The equation of motion for the $\mathrm{N}$ particle system is solved numerically by using the Verlet algorithm. ${ }^{16}$ The simulation has been performed by treating the interaction between the various components to be composed of pair-wise additive potentials. The site-site, ionsolvent and solvent-solvent potential are represented as a combination of 12-6 Lennard-Jones potential and the Coulombic interaction.

$$
U\left(r_{\alpha \beta}\right)=A_{\alpha \beta} / r^{12}-C_{\alpha \beta} / r^{6}+q_{\alpha} q_{\beta} / r .
$$

Here, $\alpha$ and $\beta$ denote a pair of interaction sites on different molecules, $r_{\alpha \beta}$ is the site-site separation, and $q_{\alpha}$ is a point charge located at site $\alpha$. The terms $A_{\alpha \beta}$ and $C_{\alpha \beta}$ are given by

$$
\begin{gathered}
A_{\alpha \beta}=4 \varepsilon_{\alpha \beta} \sigma_{\alpha \beta}^{12} \\
C_{\alpha \beta}=4 \varepsilon_{\alpha \beta} \sigma_{\alpha \beta}^{6},
\end{gathered}
$$

where $\varepsilon_{\alpha \beta}$ and $\sigma_{\alpha \beta}$ are the energy and the distance parameters in the Lennard-Jones potential. The cross
Table 1. Lennard-Jones and Columbic parameters for the solvents used in the simulations.

\begin{tabular}{lccclr}
\hline Solvent & Model & Site & $(\varepsilon / \mathrm{k}) / \mathrm{K}$ & \multicolumn{1}{c}{$\sigma / \AA$} & \multicolumn{1}{c}{$\mathrm{q} / \mathrm{e}$} \\
\hline DMSO & $\mathrm{P} 1$ & $\mathrm{~S}$ & 120 & 3.4 & 0.5400 \\
& & $\mathrm{O}$ & 35 & 2.80 & -0.5400 \\
\multirow{3}{*}{ Water } & \multirow{2}{*}{$\mathrm{SPC} / \mathrm{E}$} & $\mathrm{Me}$ & 150 & 3.80 & 0.0000 \\
& & $\mathrm{H}$ & 0.0 & 0.0 & 0.4236 \\
\hline
\end{tabular}

interactions have been obtained by using the LorentzBerthelot rules.

$$
\begin{aligned}
& \sigma_{\alpha \beta}=\left(\sigma_{\alpha \alpha}+\sigma_{\beta \beta}\right) / 2 \\
& \varepsilon_{\alpha \beta}=\left(\varepsilon_{\alpha \alpha} \cdot \varepsilon_{\beta \beta}\right)^{1 / 2} .
\end{aligned}
$$

Now, the force on each particle/site is calculated from the relation

$$
F_{\alpha}=-\sum_{\beta \neq \alpha} \partial U\left(r_{\alpha \beta}\right) / \partial \mathrm{r}_{\alpha} .
$$

In this report, we have used the SPC/E model ${ }^{17}$ of water and the P1 model ${ }^{18}$ of DMSO. The molecularity of the solvents is kept intact by using molecular dynamics with constraints on the intra-atomic distances in the molecules. The details of the solvent parameters are given in table 1 .

The P1 model of the DMSO which is used in the present work differs considerably from the P2 model ${ }^{19}$ in that the charge on $\mathrm{S}$ in P1 model $(0.54 \mathrm{e})$ is nearly four times the charge of the P2 model $(0.139 \mathrm{e})$ and the methyl groups in P1 are uncharged. The energy parameter of methyl in P1 is also twice that of the P2 model. ${ }^{18,19}$ We have already shown that the potentials of mean force are not significantly influenced by the model differences, while the P1 model gives better diffusion coefficients. We have performed extensive MD simulations on systems containing norbornyl chloride in water and DMSO. The simulation box lengths (L) and solvent densities $(\rho)$ and the solvent particle numbers (N1 and N2) are given in table 2. Over 100 ps simulations were done for each composition using the Verlet algorithm and SHAKE algorithm ${ }^{20}$ to keep the intra-atomic distances in the solvents fixed. The norbornyl chlorides are kept in the centre of the simulation cell and the solvent

Table 2. Details of the simulation boxes for DMSO and water.

\begin{tabular}{lccc}
\hline Solvent & $\begin{array}{c}\text { Number of } \\
\text { molecule }\end{array}$ & Box length/Å & Density/(g/cc) \\
\hline DMSO & 146 & 26.066 & 1.1004 \\
Water & 590 & 26.066 & 0.997 \\
\hline
\end{tabular}


molecules are arranged around it initially in a random manner and the system is equilibrated for 10 to $50 \mathrm{ps}$. Before the equilibration steps, Monte-Carlo simulations are performed to get a minimum energy configuration of the system. Generating the PMF from these simulations involves integrating the average mean force values over the range of separation between norbornyl cation and the chloride anion. ${ }^{21,22}$ The potential of mean force is obtained by integrating the mean forces as

$$
W(r)=W\left(r_{0}\right)-\int_{r_{0}}^{r} F(r) d r .
$$

We need a suitable value of $W(r)$ at $r_{0}$ to complete the integration. This is done by assuming that at a distance of $r_{0}=8 \AA, W\left(r_{0}\right)$ is given by the formula

$$
W\left(r_{0}\right)=q_{\alpha} q_{\beta} / \varepsilon r_{0},
$$

where, $\varepsilon$ is the bulk dielectric constant of the solvent. The $\mathrm{C}-\mathrm{Cl}$ distances are varied from $1.5 \AA$ to $8 \AA$ at intervals of $0.25 \AA$ in the range of $1.5 \AA$ to $5 \AA$ and at intervals of $0.5 \AA$ at larger distances. At each fixed separation of the $\mathrm{C}-\mathrm{Cl}$ distance, 100 to $200 \mathrm{ps}$ MD simulations are performed to get mean forces between the $\mathrm{C}$ atom and the $\mathrm{Cl}$ ion. These are integrated as per equation 8 to get the PMF.

\section{Results and discussion}

$A b$ initio calculations were performed to obtain the potential energy curves for the dissociation of both endo and exo 2-norbornyl chlorides at different levels of theory. In the gas phase potential energy curves, a minimum is observed at $1.75 \AA$ for both the endo and the exo 2-norbornyl chlorides. Both the minima have very similar energy but as the distance between $\mathrm{C}-$ $\mathrm{Cl}$ increases, exo is found to be more stabilized than endo. For the dissociation energy (difference between stabilized norbornyl chloride and dissociated norbornyl chloride) a reference value of the distance of $8 \AA$ is chosen. Beyond $5 \AA$, potential energy for the exo norbornyl chloride is found to be 3 to $5 \mathrm{kcal} / \mathrm{mol}$ lower than

Table 3. Dependence of the dissociation energy differences between endo and exo 2-norbornyl chlorides on the level of theory.

Level of theory

\begin{tabular}{ll}
\hline HF & 5.32 \\
B3LYP & 4.57 \\
MP2 & 3.43 \\
\hline
\end{tabular}

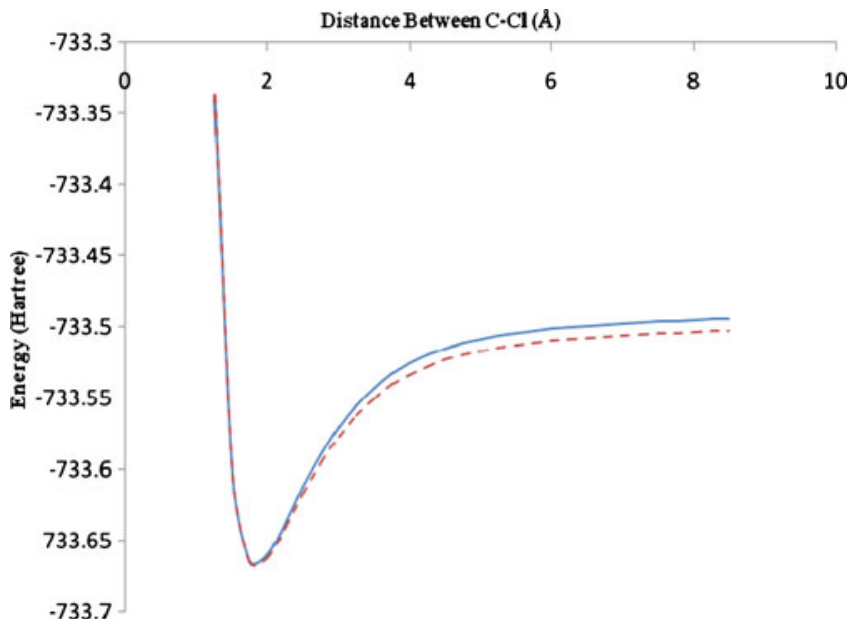

Figure 1. Potential energy diagram of endo and exo 2norbornyl chlorides in gas phase using B3LYP/6-311++G**. (continuous curve- endo, dashed curve- exo).

the corresponding value for the endo as shown in the table 3 .

Thus, in the gas phase, exo norbornyl chloride is expected to have a higher dissociation rate than endo. The potential energy curve monotonically increases to the dissociation limit for $\mathrm{C}-\mathrm{Cl}$ bond. Figure 1 shows the potential energy curves for the endo and exo dissociations.

Potential energy curves obtained from our empirical fits have a similar structure as the $a b$ initio curve obtained from the DFT calculation. The agreement from 2 to $4 \AA$ is very good (within $1-3 \mathrm{kcal} / \mathrm{mol}$ ) while it is in the range of $5-10 \mathrm{kcal} / \mathrm{mol}$ beyond $4 \AA$ (figure 2 ).

While this is by no means entirely satisfactory, these calculations will provide a starting point for further analysis of the dissociation barriers for this reaction. In

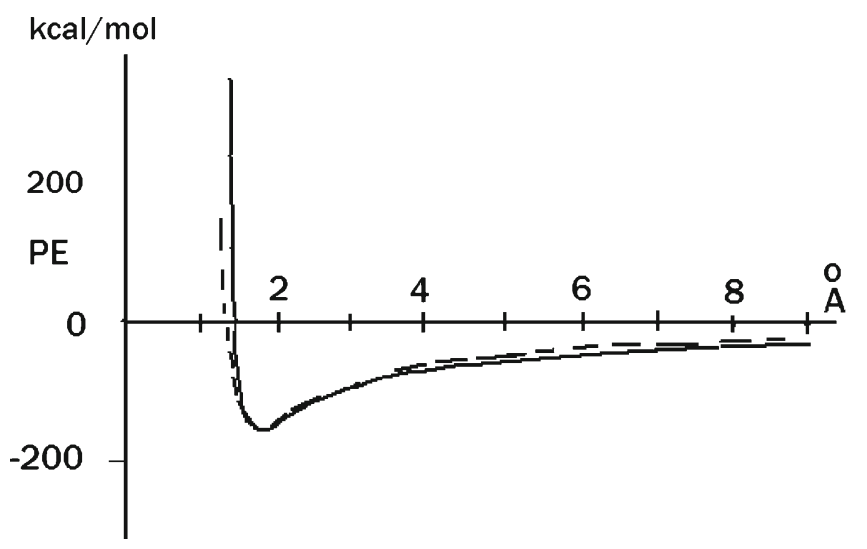

Figure 2. A comparison between an empirical potential energy curve (thick line) and the $a b$ initio potential energy curve (dashed line) obtained from DFT calculation (B3LYP/6-311++G**) for exo 2-norbornyl chloride. The $\mathrm{X}$-axis is the $\mathrm{C}-\mathrm{Cl}$ distance which is also the reaction coordinate in our simulations. 
Table 4. Lennard-Jones and Coulombic parameters for norbornyl chloride.

\begin{tabular}{lrcr}
\hline Site & $(\varepsilon / \mathrm{k}) / \mathrm{K}$ & $\sigma / \AA$ & $\mathrm{q} / \mathrm{e}$ \\
\hline $\mathrm{C}$ & 120 & 3.4 & 0.61 \\
$\mathrm{H}$ & 75 & 2.0 & -0.21 \\
$\mathrm{Cl}$ & 150 & 4.0 & -1.0 \\
\hline
\end{tabular}

our calculations we have used a fixed charge model, while in reality as the distance increases, charges on the sites also change. Thus, it is not very easy to obtain a perfect match with DFT calculations using a fixed charge model. Energy and distance parameters used in the 12-6-1 potential energy empirical equation (to obtain a fit with DFT calculation) are shown in table 4 . Parameters obtained from these calculations are used in our MD calculations.

\subsection{MD simulation in DMSO}

In figure 3, we have presented the mean forces between C-2 and chloride as a function of the distance between them.

Mean forces are calculated by averaging $F(r, t)$ over at least $100 \mathrm{ps}$. These curves have a typical accuracy of $1-2 \mathrm{kcal} \AA^{-1} \mathrm{~mol}^{-1}$. In figure 4, we have presented the PMF for endo and exo 2-norbornyl chloride dissociation in DMSO solvent at $298 \mathrm{~K}$.

In the presence of solvent, minima for both endo and exo 2-norbornyl chlorides are found to be shifted considerably from their initial locations. In gas phase, minima for potential energy curves are obtained at $1.75 \AA$

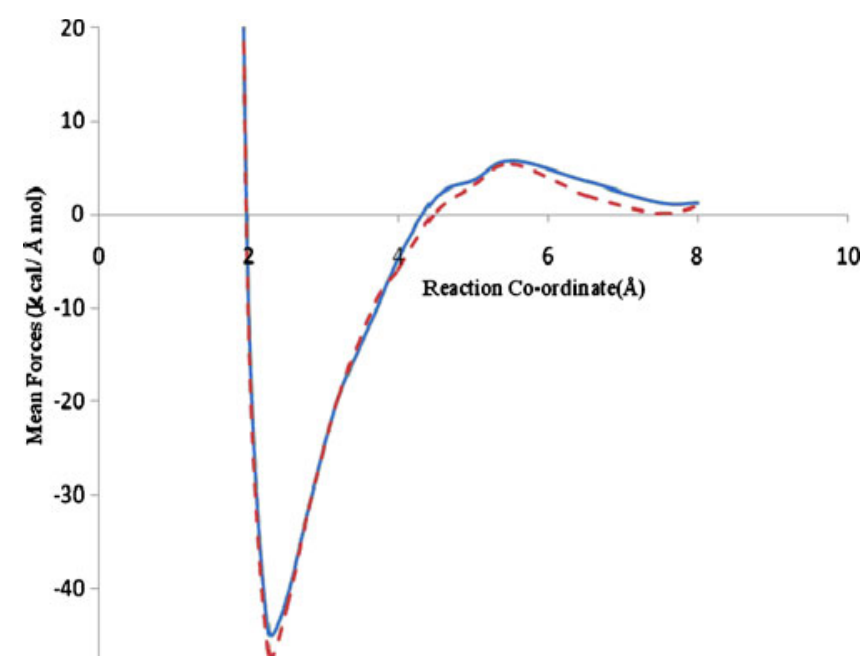

Figure 3. Mean forces vs distance for both endo (continuous curve) and exo (dashed curve) norbornyl chloride in DMSO. The reaction coordinate is the $\mathrm{C}-\mathrm{Cl}$ distance.

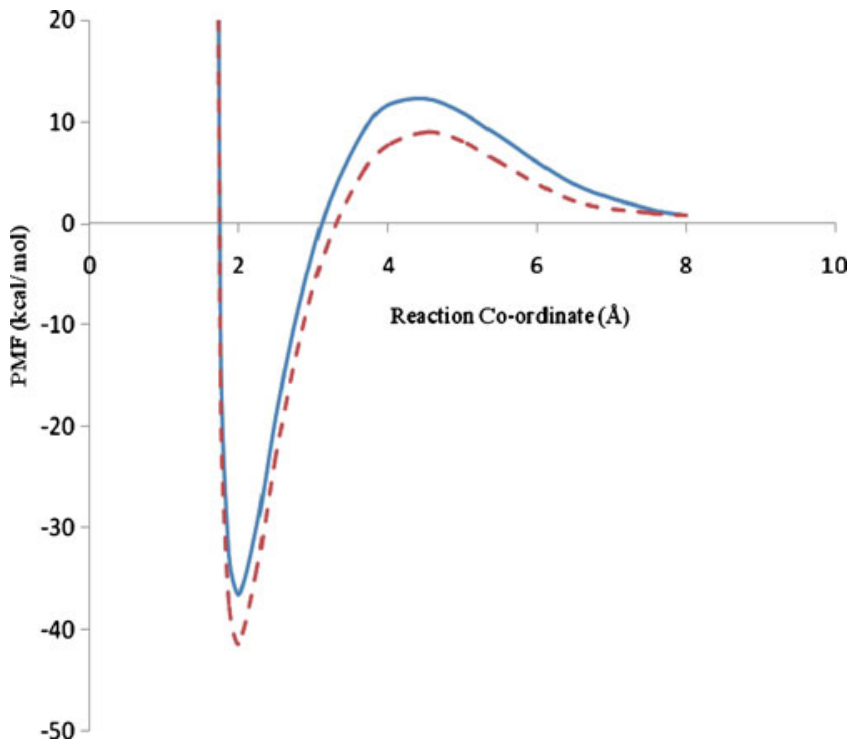

Figure 4. Potentials of mean force for both endo (continuous curve) and exo (dashed curve) norbornyl chloride in DMSO.

from our empirical formulae, while in the presence of solvent it is found to be at $2.2 \AA$. The locations of the maxima in the PMFs are at $5.5 \AA$. This could be due to the formation of the solvent separated ion pair. The activation barrier for exo is found to be $1.5 \mathrm{kcal} / \mathrm{mol}$ higher than the endo but the error bars during our calculation are also found to be in the range of 1$2 \mathrm{kcal} / \mathrm{mol}$. In DMSO, we have found an activation barrier of $50.4 \mathrm{kcal} / \mathrm{mol}$ for the exo and $48.8 \mathrm{kcal} / \mathrm{mol}$ for the endo. This barrier is caused by solvent association. This barrier is absent in the gas phase calculations (from both $a b$ initio and empirical fitted curves).

\subsection{MD simulation in water}

The mean forces $F(r)$ between the norbornyl cation and the chloride ion in the presence of pure water as a solvent are shown in figure 5 .

In figure 6, the PMFs, $W(r) s$ for both the exo and the endo norbornyl chloride are obtained by direct integration of the total mean force $F(r)$, according to equation 8 , taking $r_{0}=8.0 \AA$.

In the presence of water as solvent, the minima for both the endo and exo 2-norbornyl chlorides are found at $2.0 \AA$. The locations of the maxima in the PMFs are at $3.5 \AA$ for both the endo and the exo isomers. The gap between the minimum in the PMF and the transition state for the exo is nearly $4.0 \mathrm{kcal} / \mathrm{mol}$ higher than the endo. While the error bars during our calculation are in the range of $1.5 \mathrm{kcal} / \mathrm{mol}$ activation barriers in water are found to be $15.3 \mathrm{kcal} / \mathrm{mol}$ for endo and $19.7 \mathrm{kcal} / \mathrm{mol}$ for exo. 


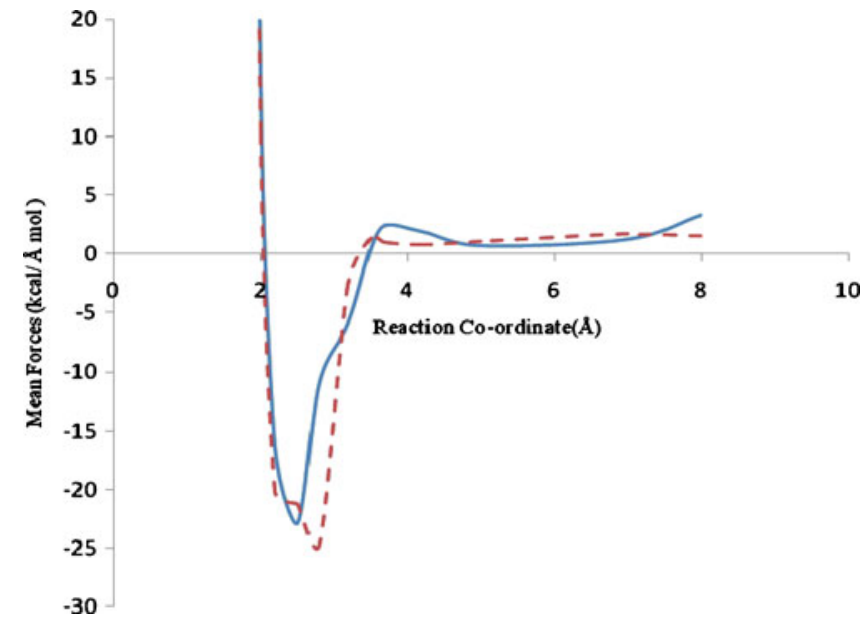

Figure 5. Mean forces $v s$ distance curve for both endo (continuous curve) and exo (dashed curve) norbornyl chloride in water. The reaction coordinate is the $\mathrm{C}-\mathrm{Cl}$ distance.

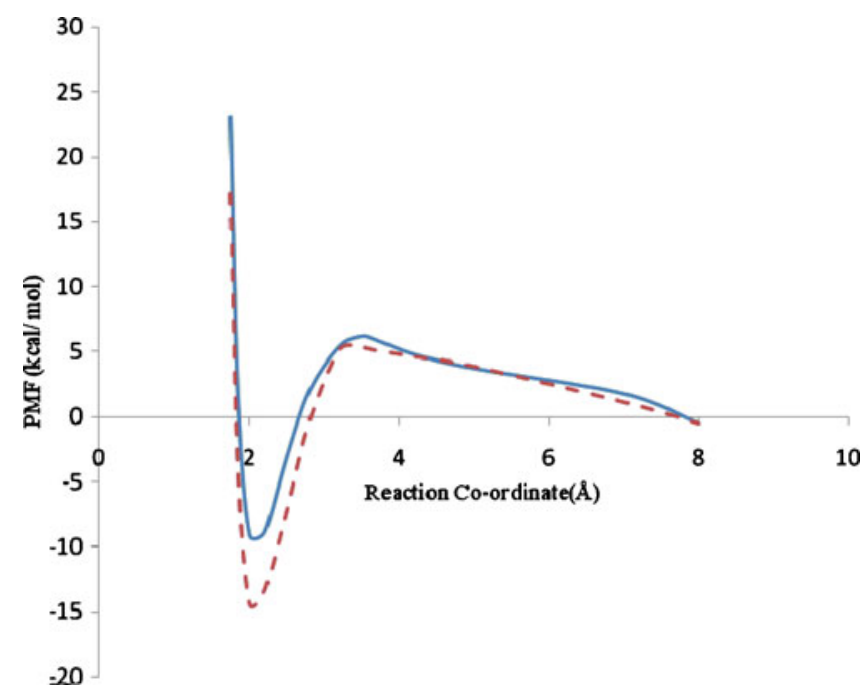

Figure 6. Potentials of mean force for both endo (continuous curve) and exo (dashed curve) norbornyl chloride in water. The reaction coordinate is the $\mathrm{C}-\mathrm{Cl}$ distance.

We would like to make a few remarks on the differences of the PMFs in water and DMSO. The depth of the minimum of the PMF in DMSO is nearly three times the corresponding depth in water. The barrier regions of the PMF extend from $3.0 \AA$ to $7.5 \AA$, the height of the barrier in DMSO being only slightly higher than that in water. Both these features seem to be primarily determined by the charge and size parameters of the solvent molecules. These features closely resemble the behaviour of the PMFs in simpler ion pair associations (such as that between sodium and chloride) in these solvents.

\section{Conclusions}

In the present paper, we have reported the potential energy curves for the dissociation of endo and exo 2norbornyl chlorides and also the effect of the two solvents on the dissociation process. On solvation, the locations of minima in the potential energy curves shift by nearly $0.25-0.4 \AA$ to the right. The locations of the barriers developed in DMSO and water are shifted from their corresponding locations of the minima by $3.2 \AA$ and $1.6 \AA$, respectively. This shift does not correspond to a complete insertion of the solvent molecule between carbon and chloride. In our ab initio calculations, dissociation of exo 2-norbornyl chloride is found to be a more facile pathway than endo dissociation. This is because the dissociation energy for the exo is about $3-5 \mathrm{kcal} / \mathrm{mol}$ lower than the corresponding value for the endo. However, MD calculations for the potential model used in the present study show an opposite trend since endo appears to be more stabilized than the exo on solvation by $2-5 \mathrm{kcal} / \mathrm{mol}$. Error bars for the potentials of mean force are of the order of $1.5 \mathrm{kcal} / \mathrm{mol}$. Several factors may be contributing to this behaviour. The chloride ion might be a much smaller ion (than species like tosylate which are used as leaving group for experimental studies) and may not capture the full essence of experimental study. Thus, we may have to explore further calculations for a larger leaving group. It is also possible that the dissociation along $\mathrm{C}-\mathrm{Cl}$ bond direction might not be the dominant pathway in the presence of solvent. Fluctuations along the pathway might also lead to a differential solvation. There could also be a significant dependence of our result on the fitted potentials used in the present MD simulations. We have used water and DMSO which represent two solvents of varying dipole moment and dielectric constant. Simulations are also under way in solvents such as wateralcohol and water acetone mixtures ${ }^{23}$ and other solvent systems wherein there are significant experimental rate constant data. To understand these details we are investigating the dynamical trajectories for the $\mathrm{C}-\mathrm{Cl}$ dissociation. Also, we plan to perform MD simulation with larger leaving group in our future study.

\section{Acknowledgements}

We thank the Indian Institute of Technology Bombay (IIT), Mumbai for the high performance computing facility and Department of Chemistry, IIT Bombay for other computational facilities. We also thank the Council of Scientific Industrial Research (CSIR), Government of India, for a Junior Research Fellowship to SCT. 


\section{References}

1. (a) Schreiner P R, Schleyer P V R and Schaefer H F 1997 J. Org. Chem. 62 4216; (b) Brown H C and Ravindranathan M 1978 J. Am. Chem. Soc. 100 1865; (c) Hartman G D and Traylor T G $1975 \mathrm{~J}$. Am. Chem. Soc. 97 6147; (d) Brown H C, Gnedin B G, Takeuchi K and Peters E N 1975 J. Am. Chem. Soc. 97 610; (e) Peters E N and Brown H C 1974 J. Am. Chem. Soc. 96 265; (f) Smith S G and Petrovich J P 1965 J. Org. Chem. 30 2882

2. Winstein S and Trifan D 1952 J. Am. Chem. Soc. 741147

3. Georing H L and Schewene C B 1965 J. Am. Chem. Soc. 873516

4. (a) Olah G A, Prakash G K S and Saunder M 1983 Acc. Chem. Res. 16 440; (b) Yannoni C S, Macho V and Myhre P C 1985 J. Am. Chem. Soc. 1075294

5. (a) Brown H C 1983 Acc. Chem. Res. 16 432; (b) Brown H C 1986 Acc. Chem. Res. 1934

6. Brown H C 1977 Nonclassical ion problem' with comment by $P V R$. Schleyer (New York: Plenum Press)

7. (a) Winstein S 1965 J. Am. Chem. Soc. 87 381; (b) Olah G A, Mateescu G D and Riemenschneider J L 1972 J. Am. Chem. Soc. 94 2529; (c) Roberts J D and Lee C C 1951 J. Am. Chem. Soc. 73 5009; (d) Schleyer P V R, Watts W E, Fort R C, Comisarow M B and Olah G A 1964 J. Am. Chem. Soc. 865679

8. (a) Stille J K and Hughes R D 1971 J. Org. Chem. 36 340; (b) Brown H C and Takeuchi K 1968 J. Am. Chem. Soc. 90 2691; (c) Benjamin B M and Collins C J 1970 J. Am. Chem. Soc. 92 3183; (d) Brown H C and Rei M H 1968 J. Am. Chem. Soc. 906216

9. (a) Schreiner P R, Severance D L, Jorgensen W L, Schleyer P V R and Schaefer H F 1995 J. Am. Chem. Soc. 117 2663; (b) Babu C S and Tembe B L 1987 Proc. Indian Acad. Sci. Chem. Sci. 98235
10. Kong J, Roy D, Lenoir D, Zhang X, Zou J J and Schleyer P V R 2009 Org. Lett. 114684

11. Werstiuk N H 2007 J. Chem. Theory Comput. 3 2258

12. Moss R A, Zheng F, Sauers R R and Toscano J P 2001 J. Am. Chem. Soc. 1238109

13. Frisch M J et al 2009 Gaussian 09, Revision A.02 (Wallingford, CT: Gaussian, Inc.)

14. (a) Becke A D 1988 Phys. Rev. A 38 3098; (b) Becke A D 1993 J. Chem. Phys. 98 5648; (c) Lee C, Yang W and Parr R G 1988 Phys. Rev. B 37785

15. (a) Head-Gordon M, Pople J A and Frisch M J 1998 Chem. Phys. Lett. 153 503; (b) Frisch M J, Head-Gordon M and Pople J A 1998 Chem. Phys. Lett. 166 275; (c) Frisch M J, Head-Gordon M and Pople J A 1998 Chem. Phys. Lett. 166281

16. Verlet L 1967 Phys. Rev. 15998

17. Berendsen H J C, Grigera J R and Straatsma T P 1987 J. Phys. Chem. 916269

18. Pradhan E and Tembe B L 2010 Proc. Comput. Sci. 1 1165

19. Rao B G and Singh U C 1990 J. Am. Chem. Soc. 112 3803

20. (a) Allen M P and Tildesley D J 1987 Compuater simulation of liquids (Oxford: Oxford Science Publication); (b) Gunsteren W F V and Berendsen H J C 1977 Mol. Phys. 341311

21. Das A K 1998 Simulation studies on the sodium chloride ion pair in DMSO and water-DMSO mixtures; Ph.D. Thesis, IIT Bombay, Mumbai, India

22. Madhusoodanan M 1994 Studies in the molecular fluids: carbon disulphide, acetonitrile, dimethyl sulphoxide and ion pairs in dimethyl sulphoxide; Ph.D. Thesis, IIT Bombay, Mumbai, India

23. Brown H C and Gundu Rao C 1980 J. Org. Chem. 45 2113 